Research Article

\title{
Evaluation of Precipitation Forecast of System: Numerical Tools for Hurricane Forecast
}

\author{
José C. Fernández-Alvarez $\mathbb{D}^{1},{ }^{1}$ Albenis Pérez-Alarcon $\mathbb{D}^{1},{ }^{1}$ Alfo J. Batista-Leyva, ${ }^{2}$ \\ and Oscar Díaz-Rodríguez ${ }^{3}$ \\ ${ }^{1}$ Departamento de Meteorología, Instituto Superior de Tecnologías y Ciencias Aplicadas, Universidad de la Habana, La Habana, \\ Havana 10400, Cuba \\ ${ }^{2}$ Departamento de Física Atómica y Molecular, Instituto Superior de Tecnologías y Ciencias Aplicadas, Universidad de la Habana, \\ La Habana, Havana 10400, Cuba \\ ${ }^{3}$ Centro de Física de la Atmósfera, Instituto Cubano de Meteorología, La Habana, Havana 10400, Cuba
}

Correspondence should be addressed to José C. Fernández-Alvarez; jcfernandez@instec.cu

Received 20 April 2020; Revised 11 July 2020; Accepted 19 July 2020; Published 5 August 2020

Academic Editor: Stefania Bonafoni

Copyright (C) 2020 José C. Fernández-Alvarez et al. This is an open access article distributed under the Creative Commons Attribution License, which permits unrestricted use, distribution, and reproduction in any medium, provided the original work is properly cited.

\begin{abstract}
Heavy rainfall events, typically associated with tropical cyclones (TCs), provoke intense flooding, consequently causing severe losses to life and property. Therefore, the amount and distribution of rain associated with TCs must be forecasted precisely within a reasonable time to guarantee the protection of lives and goods. In this study, the skill of the Numerical Tool for Hurricane Forecast (NTHF) for determining rainfall pattern, average rainfall, rainfall volume, and extreme amounts of rain observed during TCs is evaluated against Tropical Rainfall Measuring Mission (TRMM) data. A sample comprising nine systems formed in the North Atlantic basin from 2016 to 2018 is used, where the analysis begins $24 \mathrm{~h}$ before landfall. Several statistical indices characterising the abilities of the NTHF and climatology and persistence model for rainfalls (R-CLIPER) for forecasting rain as measured by the TRMM are calculated at 24,48 , and $72 \mathrm{~h}$ forecasts for each TC and averaged. The model under consideration presents better forecasting skills than the R-CLIPER for all the attributes evaluated and demonstrates similar performances compared with models reported in the literature. The proposed model predicts the average rainfall well and presents a good description of the rain pattern. However, its forecast of extreme rain is only applicable for $24 \mathrm{~h}$.
\end{abstract}

\section{Introduction}

Tropical cyclones (TCs) are among the most devastating atmospheric phenomena, as they result in strong surface winds, tornadoes, storm surges, and heavy rainfall events. Heavy rainfall events are distributed over wide areas and can cause flash flooding, thereby resulting in human and economic losses. It has been reported that approximately $60 \%$ of human deaths caused by hurricanes in the USA were related to flash flooding $[1,2]$. In Cuba, Hurricane Flora (1963) caused approximately 2000 casualties due to heavy persistent rains [3]. These facts highlight the importance of an accurate forecast of the distribution and amount of rain during the interaction of a TC with land.
The rain pattern of a TC depends on different factors, namely, its internal dynamics, the synoptic situation around the cyclone, and its translational speed, which provoke azimuthal asymmetries [4]. It has been reported that vertical wind shear creates asymmetries in the inner-core field rainfall distribution pattern $[5,6]$. The interaction of the storm with the Earth surface, as well as the available humidity, and intensity of the cyclone significantly affect the distribution and amount of rain [7]. For instance, a close relationship between precipitation distribution and thermodynamical symmetry has been discovered in the evolution of Hurricane Edouard [8]. In recent years, these factors have been incorporated in numerical models to perform a quantitative forecast of the track, intensity, and precipitation 
of TCs. The incorporation of a better physical representation of processes associated with hurricanes, as well as different parameterization schemes, allows a higher precision of the forecast of these elements with better spatial and time resolutions $[9,10]$. However, most previous studies have focused on forecasting the intensity and track of cyclones; fewer studies have focused on precipitation forecast. For example, DeMaria and Tuleya [11] evaluated the precipitation forecast of model Geophysical Fluid Dynamics Laboratory (GFDL) in the North Atlantic basin (NATL) for cyclones affecting mainland USA. Marks and DeMaria [12] developed an equivalent of the climatology and persistence model for rainfalls (R-CLIPER), where the intensity of the climatological precipitation is accumulated in the storm's track. This model is widely used as a benchmark to evaluate other precipitation forecast techniques.

Marchok et al. [7] reviewed and applied different validation schemes of the most frequently used forecast models regarding their ability to predict different aspects of rainfall, i.e., its distribution in time and space, its mean rainfall, and extreme rains observed in TCs. The validation was performed with all the TCs land-falling in the USA from 1998 to 2004. A similar study for the North Indian Ocean was performed as well [13], where the precision of precipitation forecast of several global models used in the area was evaluated through comparison with TRMM-3B42 data. The sample was composed of nine TCs that formed in the North Indian Ocean from 2010 to 2013. Those researchers discovered that, although the performances of some models were similar to the observations, no single model predicted all the observed features equally well. Nevertheless, the TRMM data must be used cautiously owing to the demonstrated underestimation of heavy rainfalls in mountainous regions [14] and other drawbacks, which will be discussed in the following.

In Cuba, two operational systems are used for precipitation forecast. In 2015, Sierra et al. [15] proposed a configuration derived from the Weather Research and Forecasting model (WRF-ARW), known as Sistema de Pronóstico Inmediato. The main objective of the configuration was to perform a short-term prediction. They discovered the largest precipitation forecast errors from July to November, coinciding with the hurricane season in the NATL; therefore, they concluded that none of the tested configurations correctly forecasted the rainfall associated with TCs. The other operational system, named Sistema de Pronóstico Numérico Océano Atmósfera, combines the WRF-ARW with two sea wave models (WW3 and SWAN) and an oceanic circulation model (ROMS) $[16,17]$. The authors found the best forecast ability for rainfall thresholds above $5 \mathrm{~mm}$ /day during the months of April, May, and June, out of the cyclone season. Currently, an evaluated operational model demonstrating good rain forecasts for TCs does not exist.

The Department of Meteorology of the Higher Institute of Applied Technologies and Sciences, University of Havana developed an operational model that can be used to forecast precipitation. It is known as the Numerical Tool for Hurricane Forecast (NTHF) [18], which incorporates the option of a GFDL vortex tracker. It is a program that loads model forecasts in the GRIB/NetCDF format, objectively analyses data to provide an estimate of the vortex centre position (latitude and longitude) and tracks the storm for the duration of the forecast. It includes parameterization schemes to describe the physics related to the development and intensification of hurricanes.

Furthermore, it uses the atmospheric component of the HWRF3.9 model, which is specifically designed to be used for forecasting TCs $[19,20]$. It has been demonstrated that this system can forecast the track of TCs, particularly Category 4 and 5 hurricanes in the Saffir-Simpson scale [18]. Regarding intensity, the system has forecasting abilities of cyclonic systems from depressions to Category 3 hurricanes, with discrete results for Category 4 and 5 hurricanes. Although the model can forecast rainfall [20], it has not been evaluated for Cuba and the Inter-American Oceans. The aim of this study is to evaluate the abilities of the NTHF system for forecasting rainfall associated with TCs, as reported by the TRMM. The system demonstrated excellent predictions of the average rainfall and a good description of the rain pattern; however, its forecast of extreme rain was only applicable for $24 \mathrm{~h}$.

\section{Observational and Modelled Data}

2.1. HWRF and R-CLIPER Models. The NTHF has been implemented and is operational at the Department of Meteorology of the Higher Institute of Technologies and Applied Sciences of the University of Havana. Its aim is to forecast the evolution of TCs formed in the NATL, particularly in intercontinental seas. The model uses the HWRF as a dynamic core for the solution of a system of primitive equations. The computational algorithms guarantee the initialisation of the model during operational runs with official information from the National Hurricane Centre (NHC) and the forecast outputs of the Global Forecasting System (GFS). Furthermore, it contains algorithms for postprocessing the results. The forecasts extend for $120 \mathrm{~h}$ durations, consistent with the time period of official NHC forecasts. Figure 1 shows the NTHF block diagram [18].

The configuration of the model for this study was based on the recommendations reported in [21, 22] for the operational runs of the HWRF in the National Centre for Environmental Prediction (NCEP). Experiments were performed with bidirectional interactive nested domains of $27-9 \mathrm{~km}$ resolution. The external domain was located in the centre of the storm, whereas the internal domain tracked the centre of the storm during the integration of the model using a movable grid. Figure 2(a) shows the nested domains.

All performed simulations were initialised at 0000 UTC with the outputs of the GFS at a $0.5^{\circ}$ resolution, as obtained from https://nomads.ncdc.noaa.gov/data/gfs4. They were performed for a forecast period of $72 \mathrm{~h}$. The boundary conditions were updated every $6 \mathrm{~h}$, and the temporary integration step was $69 \mathrm{~s}$ for the $27 \mathrm{~km}$ domain; meanwhile, the temporary integration of the internal domain was $1 / 3$ of the external domain temporary step. Table 1 shows the fundamental aspects of the configuration used. 


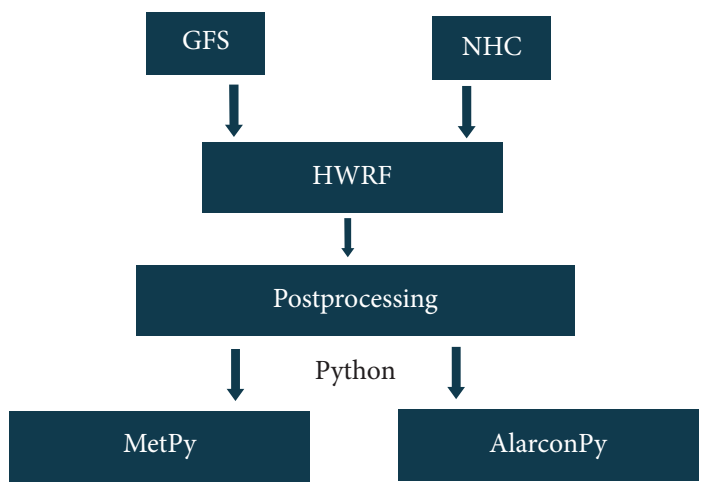

Figure 1: NTHF block diagram. MetPy and AlarconPy are Python packages for the treatment and management of meteorological data.

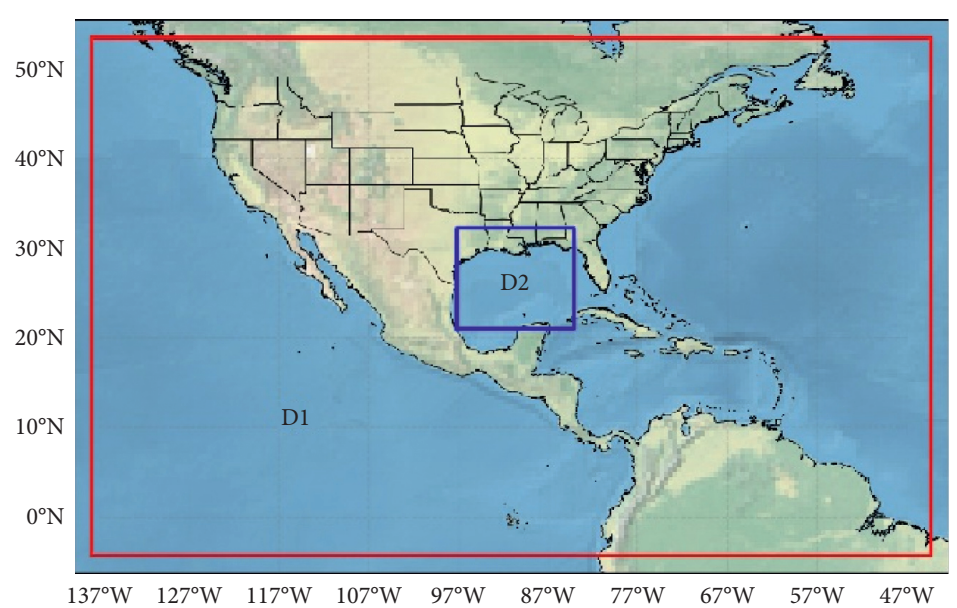

(a)

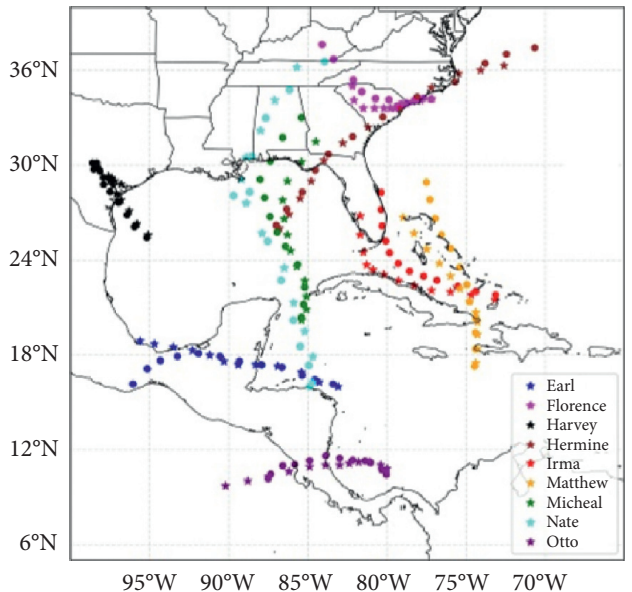

(b)

Figure 2: Study area and study cases. (a) Movable nested domains used in the implementation. The inner domain D2 moves with the storm. (b) Cyclones used for evaluation. Circles represent the predicted track, whereas stars represent real trajectories. Most of the selected cyclones developed in the Caribbean Sea and the Gulf of Mexico.

TABle 1: Configuration used in the model.

Vertical resolution

Parameterizations of longwave radiation

Parameterization of shortwave radiation

Cumulus parameterization

Microphysics parameterization

Parameterization of the planetary boundary layer

Surface-layer parameterization

Land model

Vortex tracker

Vortex relocation

Coupling with the ocean model
32 vertical levels

Rapid radiative transfer model for general circulation models (RRTMG) Rapid radiative transfer model for general circulation models (RRTMG) Scale-aware simplified Arakawa-Schubert Ferrier-Aligo scheme HWRF planetary boundary layer HWRF surface-layer scheme Noah land surface model GFDL vortex tracker No No
This configuration has certain limitations associated with the noncoupling of the ocean model. Therefore, the model uses a static sea surface temperature, rendering it impossible to account for changes in temperature during the model integration, thereby affecting the calculated intensity [23]. Another shortcoming of the system is that it does not use vortex relocation, thereby affecting some evaluation parameters, as discussed below. For a description of the parameterizations used and a discussion of the shortcomings of the configuration, see [18].

Despite the limitations mentioned above, the NTHF is an alternative to the NOAA HWRF system [22]. It can be implemented in centres without high computational resources for operational use in TC forecasts in the NATL 
basin. In addition, the implementation of the NTHF as an operating system in the NATL allows the meteorological offices of countries in Central America and the Caribbean to develop graphic products, such as monitoring cones as well as intensity and monitoring forecasts, by incorporating the results of all numerical forecasting models from https://ftp. nhc.noaa.gov/atcf/com/. Furthermore, NTHF would become a powerful tool for the scientific research of hurricanes in these countries, as an alternative to the NOAA HWRF system, because the implementation of the HWRF model requires high computational resources.

As a baseline, the R-CLIPER [12] model was implemented at the Department of Meteorology. The precipitation field was calculated using the best-track and maximum speed $72 \mathrm{~h}$ forecasts, for the latitudes and longitudes of the TRMM data mesh. Calculations were performed with a spatial resolution of $27 \mathrm{~km}$ and at $6 \mathrm{~h}$ time intervals.

2.2. TRMM Data Used in Evaluation. The study area was the NATL basin. It was selected because it constitutes a region with intense cyclonic activity. The cyclonic systems are likely to affect the Caribbean Islands as well as Central and North Americas. In addition, the study area is susceptible to developing maximum intensities owing to high sea surface temperatures in areas of the Caribbean Sea, the West Atlantic, and the Gulf of Mexico, thereby increasing the likelihood of heavy rainfall events, large accumulations, and consequently loss of human lives and economic damages.

To evaluate the NTHF configuration, nine TCs were selected (see Table 2). All of them were formed in the NATL, and most of them in the Intra-American Seas. The selection criterion was based on selecting TCs that arrived on land during the 2016, 2017, and 2018 study periods. In addition, the runs were initialised $24 \mathrm{~h}$ before landfall at $0000 \mathrm{UTC}$ to provide forecasts with the highest accuracies of the accumulated rainfall in that time interval. Cyclones with different characteristics and intensities were included, i.e., tropical storms and hurricanes with various categories, to verify the general performance of the operational configuration. Figure 2(b) shows the trajectories predicted by the NTHF and the best track for all study cases.

Owing to the development of satellite observation systems, the estimation of precipitation has been widely applied in weather and climate research, in particular for the study of rain in TCs over oceans and arriving on land. Although rain gauge data measurements are available, they are often not sufficiently dense or do not have an appropriate spatial distribution in important regions, mainly on land and near coastal areas. In addition, weather radars provide good spatial and temporal resolutions, but the area included is limited. Therefore, satellite precipitation estimates are suitable for studying rainfall characteristics, especially over oceans and coasts, where surface observations are limited [24].

The efficacy of using TRMM data in describing the characteristics of systems that reach the land has been evaluated in several studies [25-27]. These studies indicated that TRMM-3B42 data can reasonably represent the
TABLE 2: Study cases.

\begin{tabular}{lccc}
\hline Name & Category & Study period & Max. category \\
\hline Earl & Tropo UTC & & 1 \\
Hermine & Tropical storm & 3-6 August 2016 & 1-4 September 2016 \\
Matthew & Hurricane & 4-7 October 2016 & 5 \\
Otto & Tropical storm & 23-26 November 2016 & 3 \\
Harvey & Hurricane & 25-28 August 2017 & 4 \\
Irma & Hurricane & 8-11 September 2017 & 5 \\
Nate & Tropical storm & 6-9 October 2017 & 1 \\
Florence & Hurricane & 14-17 September 2018 & 4 \\
Michael & Hurricane & 8-11 September 2018 & 5 \\
\hline
\end{tabular}

The second column gives the intensity at the beginning of the observation period.

distribution of rainfall compared with rain gauge data and radar observations. However, it has been reported that TRMM-3B42 data underestimate moderate and heavy rainfalls but overestimate light precipitation [27]. Meanwhile, TRMM data collected at daily to monthly intervals are often underestimated for mountainous regions in tropical and middle latitude mountain systems $[28,29]$. Hence, it is advisable to consider the corrections introduced in [14]. Furthermore, TRMM-3B42 has a lower detection probability and lower false-alarm rates than other products in both warm and cold seasons for North America [30]. TRMM data are available on a grid with a spatial resolution $0.25^{\circ} \times 0.25^{\circ}$. They are the result of the combination of TRMM and other satellite estimates, as the Multisatellite Precipitation Analysis (TMPA). It is a trihour product that provides information from $50^{\circ} \mathrm{N}$ to $50^{\circ} \mathrm{S}$, available at http://www.pmm.nasa.gov/ data-access/downloads/trmm.shtml/.

\section{Methodology}

To evaluate the performance of each model in predicting observational data, a set of statigraphs from Brown et al. was used [31]. Rain field data simulated by models NTHF and R-CLIPER were statistically compared with the satellite observations of the TRMM. Three important elements in the forecast of TC precipitation were considered: ability to match rainfall patterns around the centre (pattern matching), ability to match average and distribution of rainfall volume (mean rainfall distribution), and ability to reproduce the largest rain values that are typically related with TCs (extreme rain prediction). The R-CLIPER model was used as a reference and comparison for all study cases.

For the interpolation of the NTHF precipitation fields to the latitudes and longitudes of the TRMM, the nearest neighbour method was used [32], in which a $600 \mathrm{~km}$ grid was selected with respect to grid's centre to avoid the inclusion of rain fields associated with another type of synoptic systems $[7,13]$. A radius measuring $5^{\circ}(\sim 555 \mathrm{~km})$ around the centre has already been tested by Englehart and Douglas [33], who demonstrated that the distance between the centre of a TC and the outer edge of its cloud shield was between 500 and $600 \mathrm{~km}$ for $90 \%$ of the cases. Moreover, Larson et al. [34] conducted sensitivity tests using radii varying between $2.5^{\circ}$ and $7.5^{\circ}$; they discovered that radii larger than $5^{\circ}$ excluded 
most TC rainfall. Zhan et al. [35] used a radius of $500 \mathrm{~km}$, which accounted for rainfall located from the inner core to adjacent rainbands. On the contrary, Rios Gaona et al. [36] used a radius of $1000 \mathrm{~km}$ to determine rainfall associated with a large number of TCs (166) for two years in different basins. However, in the presentation of the rain data, the graphs were always limited to a radius of approximately $600 \mathrm{~km}$, showing rainfall intensities $\leq 1 \mathrm{~mm} / \mathrm{h}$ at a radius of $600 \mathrm{~km}$ in all cases. Hence, it may be concluded that the inclusion of data for larger radii did not significantly improve the quality of the results.

Once rain fields have been calculated, they were used to calculate a group of statigraphs, which are described in the next paragraph. Using the statigraphs, the forecast skill indices of both models were obtained and compared to assess the performance of the NTHF. Next, we define the statigraphs and their associated indices, where $x_{i}$ represents an observed datum, $y_{i}$ the corresponding forecast value, and $n$ the sample size.

3.1. Rain Pattern Matching. To determine the ability of the system in predicting rainfall distribution, two statigraphs were used. First, the correlation pattern was measured as the Pearson correlation coefficient between the simulated and observed values in all grid points. Next, the Equitable Threat Score (ETS), which is the percent of local rainfall correctly predicted by the system, was calculated. These two statigraphs are dependent on the geographical location of the rain and hence sensitive to the track error. The points were selected up to $600 \mathrm{~km}$ away from the centre of the real track for the NTHF, R-CLIPER, and TRMM. The Pearson correlation coefficient is an index that measures the degree of linear correlation between two related variables. The closer the correlation coefficient of two variables to 1 , the more similar is the behaviour between both variables. For a sample, it can be calculated as follows:

$$
r_{p}=\frac{\sum_{i=1}^{n}\left(x_{i}-\bar{x}\right)\left(y_{i}-\bar{y}\right)}{\sqrt{\sum_{i=1}^{n}\left(x_{i}-\bar{x}\right)^{2}} \sqrt{\sum_{i=1}^{n}\left(y_{i}-\bar{y}\right)^{2}}} .
$$

$\bar{x}=\left(\sum_{i=1}^{n} x_{i} / n\right)$ and $\bar{y}=\left(\sum_{i=1}^{n} y_{i} / n\right)$ are the average values of the observed values and forecasts, respectively.

The second statigraph is related to the correct forecast of rainfall above a specified threshold in different grid points. In this case, the variable is dichotomous; therefore, it is convenient to construct a contingency table. To determine the accuracy of the forecast, the results are categorized into four groups:

(i) $H$ : the number of times the system forecasts a precipitation event above a specified threshold, and it occurs (known as hits)

(ii) $M$ : the number of times the event was not forecast but it occurred (missing)

(iii) FA: the number of times the event was forecast but did not occur (false alarms) (iv) CR: the number of times the event was not forecast, and it did not occur (correct rejection)

The contingency table (Table 3) shows a comparison of the NTHF predictions and TRMM observations; YES means an event (predicted or observed) above a specified threshold, whereas NO means a nonoccurrence.

The ETS, also known as the Gilbert skill score, is calculated based upon the contingency table. It represents the fraction of all observed and predicted events that were correctly diagnosed and considers the possibility of a correct forecast that occurs by chance. It presents the difficulty that does not distinguish the sources of error. The index is calculated as follows:

$$
\text { ETS }=\frac{H-H_{\text {random }}}{H+M+\text { FA }-H_{\text {random }}},
$$

where $H_{\text {random }}$ is an evaluation of the number of positive forecasts that occurred by chance, which can be calculated as

$$
H_{\text {random }}=\frac{N_{\mathrm{obs}(\mathrm{Yes}) *} N_{\mathrm{fsc}(\mathrm{Yes})}}{N},
$$

where $N_{\mathrm{obs}(\mathrm{Yes})}=H+M$ is the total number of rain occurrences, $N_{\mathrm{fsc}(\mathrm{Yes})}=H+\mathrm{FA}$ is the total number of rain forecasts, and $N$ is the total number of events. The closer the score is to 1 , the better is the skill.

3.2. Mean Rainfall and Rain Flux Distribution. The average rainfall and rain volume distributions are good indicators to evaluate the intensity of rain (heavy, moderate, and light) and the limitations of a model in predicting it. In our analysis for the NTHF and R-CLIPER, all grid points within $600 \mathrm{~km}$ from the centre of the forecast track (relative track) were considered, while for the TRMM the $600 \mathrm{~km}$ radius was centred in the real track.

The radial distributions, volume, root mean square error (RMSE), mean absolute error (MAE), and bias were determined for both models. Subsequently, the probability distribution functions (PDFs) and cumulative distribution functions (CDFs) were constructed. The 50th percentile of the $\mathrm{CDF}$ is important to determine the central value of the distribution such that the behaviour of the remaining rainfall thresholds that are greater than the median can be ascertained. In addition, a more detailed frequency distribution analysis of the rain behaviour was performed based on bands of $100 \mathrm{~km}$ to an outer radius of $600 \mathrm{~km}$. The definitions of the magnitudes involved are presented below.

The MAE is a measure of the deviation of forecast values from observed values. It is calculated as follows:

$$
\operatorname{MAE}=\frac{\sum_{i=1}^{n}\left|x_{i}-y_{i}\right|}{n} .
$$

The closer the MAE values are to zero, the more accurate is the forecast. 
TABle 3: Contingency table (NTHF-TRMM).

\begin{tabular}{llcc}
\hline & & \multicolumn{2}{c}{ TRMM } \\
& & YES & NO \\
\hline \multirow{2}{*}{ NTHF } & YES & $H(Y Y)$ & FA (YN) \\
& NO & $M(\mathrm{NY})$ & CR (NN) \\
\hline
\end{tabular}

The root mean square error (RMSE) enables the magnitude of the deviation of the forecast values from the observed values to be quantified. It is calculated as follows:

$$
\mathrm{RMSE}=\sqrt{\frac{\sum_{i=1}^{n}\left(x_{i}-y_{i}\right)^{2}}{n}} .
$$

When the RMSE $=0$, the forecast is perfect. The RMSE is a measure of accuracy in the forecast.

Bias provides the difference between the estimated and observed values, considering the sign of the deviations as follows:

$$
\text { BIAS }=\frac{\sum_{i=1}^{n}\left(x_{i}-y_{i}\right)}{n} .
$$

A simulation is good when the BIAS values are close to zero. Unlike the MAE, whose magnitude is always nonnegative, the BIAS can assume both positive and negative values, thereby allowing one to determine if an under- or overestimation had occurred in the forecast.

3.3. Extreme Rain Prediction. It is important to assess if the system can reproduce extreme rain events. In this case, the 95th percentile of the cumulative frequency distribution was analysed. To calculate it, the relative track was used for the NTHF and R-CLIPER, whereas the TRMM was centred in the actual track. In addition, the same method was applied to the $0-100 \mathrm{~km}$ and $300-400 \mathrm{~km}$ bands.

3.4. Skill Indices. The skill indices in the quantitative precipitation forecast (QPF) proposed by Marchok et al. [7] are used to determine the ability of the model. The closer the index to 1 (or 100\%), the better the performance of the model.

3.4.1. Pattern Matching. This index corresponds to the ability of the model in reproducing rain patterns. It is obtained by averaging the average ETS determined for all precipitation thresholds and the average correlation coefficient:

$$
\mathrm{PM}=\frac{\overline{r_{p}}+\overline{\mathrm{ETS}}}{2} .
$$

3.4.2. Average Rainfall and Its Distribution. This corresponds to the ability of the model in representing the average rainfall and its distribution. It can be calculated using the average of the mean rainfall error index (MREI) and the median value index in the cumulative frequency distribution (CDF-MVI).
The MREI index is expressed as

$$
\text { MREI }=\frac{1}{n} \sum_{i=1}^{n}\left(1-\frac{\left(\bar{R}_{f i}-\bar{R}_{o i}\right)}{R_{\max }}\right),
$$

where $n$ is the number of radial intervals; $\bar{R}_{f i}$ and $\bar{R}_{o i}$ are the predicted and observed rain averages for the $i$ radial interval, respectively. These were determined for 20 intervals from 30 to $600 \mathrm{~km}$ around the centre of the storm by averaging the rain at each grid point located in each interval. $R_{\max }$ is the maximum observed rain in some bands corresponding to the system.

The CDF-MVI is expressed as

$$
\mathrm{CDF}-\mathrm{MVI}=\left(1-\left|R_{f 50 \%}-R_{o 50 \%}\right|\right),
$$

where $R_{f 50 \%}$ and $R_{050 \%}$ are the rain thresholds corresponding to the $50 \%$ percentile in the CDF for the model and observations, respectively. In this formulation, the index is high (low) when the average differences between the rain thresholds are small (large). If the difference exceeds 1 inch $(25.4 \mathrm{~mm})$, the index value is zero.

3.4.3. Extreme Rain Amount. The maximum value index (CDF-MI) represents the ability of the model in forecasting extreme rain observed in TCs. It is calculated as follows:

$$
\mathrm{CDF}-\mathrm{MI}=1-\left(\mathrm{CDF}_{95 \%}-95\right)^{2} \text {, }
$$

where CDF 95\% is the percentile in the CDF of the model, corresponding to the precipitation threshold in the $95 \%$ percentile for the CDF of the observations.

Table 4 shows a summary of the indices and the dependence with the track error. A library (QPF-verif1.0) Python 3.7 based on Anaconda3 packages [37] was implemented for processing the TRMM data and the outputs of the NTHF system and R-CLIPER model, as well as for the representation of the results.

\section{Results and Discussion}

4.1. Errors in Track Prediction for Study Cases. It is mandatory to evaluate the accuracy of the track forecast for TCs because it is a key parameter that affects the results of the precipitation forecast, both in the interest region and in concrete locations. For verification, the HURDAT2 database was used. This dataset contains six-hour text information regarding the location, maximum winds, minimum central pressure, and size of all known tropical and subtropical cyclones [38]. It is available at https://www.nhc.noaa.gov/ data/\#hurdat. Figure 3 shows the errors in track forecast for the study cases, the average error for the sample, and the average error of the NHC for all the TCs studied.

As shown, the average track forecast error for the nine study cases was similar to that of the NHC until approximately $42 \mathrm{~h}$. The difference increased considerably from 48 to $72 \mathrm{~h}$, exceeding $100 \mathrm{~km}$ at the end of the forecast period. Most of the hurricanes considered have similar time evolution of the forecast error in the first $42 \mathrm{~h}$. This enables us to 
TABLE 4: Indices calculated to evaluate predictive skills of the system.

\begin{tabular}{|c|c|c|c|c|}
\hline Index & Depends on track error & Pattern & Mean/volume & Maximum value \\
\hline Large-scale ETS & Yes & $x$ & & \\
\hline Pattern correlation & Yes & $x$ & & \\
\hline Mean rainfall error index & Yes & & $x$ & \\
\hline Track-relative CDF median value & No & & $x$ & \\
\hline Track-relative CDF percentage in 95th percentile & No & & & $x$ \\
\hline
\end{tabular}

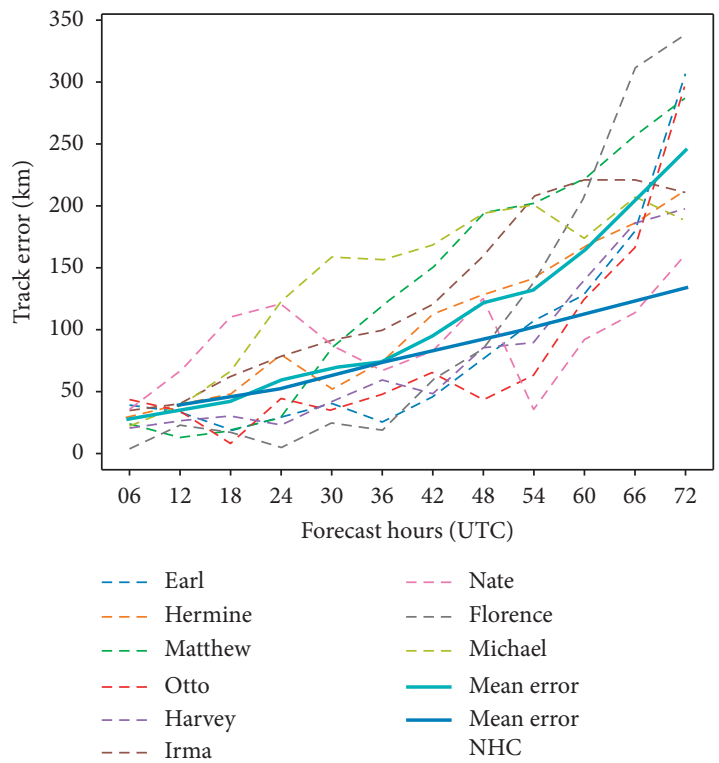

FIgURE 3: Errors in track forecast. Thick light blue continuous line represents average track error for all TCs investigated in this study. Thick dark blue continuous line represents average track error for the same TCs studied by the NHC, and discontinuous lines represent track forecast error for each TC considered herein.

associate the errors in the precipitation forecast for these time periods with the track error.

4.2. Comparison of Model Forecast and TRMM Output. In Figure 4, a comparison of the accumulated precipitation forecasted using the NTHF (Figure 4(a)) for Hurricane Irma and the accumulated values obtained from the TRMM (Figure 4(b)) in the first six forecast hours is shown. Both results were obtained at 1800 UTC, September 8, 2017, and the accumulated precipitation in a duration of $6 \mathrm{~h}$ was considered. These values are the approximate data used to determine the statigraphs and indices for evaluating the forecast quality. For instance, to determine the BIAS (see (6)), the value of the difference between the corresponding grid points of the two maps is calculated and averaged. Figure 4(c) shows the distribution of the difference based on the considered area. The stars represent the centre of the storm according to the best track (black star) and the track predicted by the NTHF (red star). In Figure S1 of the Supplementary Materials, the accumulated precipitation forecasted by R-CLIPER for the same hurricane, date, and time is shown. By adding the precipitation values for consecutive time intervals, the accumulated values for longer forecast periods can be calculated. Next, we discuss the average of the values accumulated from the sample.

\subsection{Rain Pattern Matching}

4.3.1. Pattern Correlation (PC). In Figure 5, the correlation coefficients (calculated using (1)) between the rain distribution predicted by the NTHF and R-CLIPER with the values provided by the TRMM are shown. The results spanned from 6 to $72 \mathrm{~h}$ of forecast, with a $6 \mathrm{~h}$ interval. It is clear that the NTHF achieved correlation coefficients exceeding 0.6 for all forecast intervals. The PC increased with time, reaching its maximum values from 18 to $48 \mathrm{~h}$, and then decreased. The initial increment appeared to be provoked by the self-adjustment of the fields, whereas the final decrement was governed by the increment in the track forecast error (see Figure 3). Regarding the R-CLIPER, its $r \geq 0.5$; however, in the time interval from 48 to $60 \mathrm{~h}$ of the forecast time, its $r \geq 0.6$, which is equal to that of the NTHF. This is likely due to the weakening of the cyclones after a landfall, which improves the R-CLIPER forecasting capabilities based on a mean representation. Both forecasts improved with time and exhibited a final decrement. In general, NTHF performed better than R-CLIPER.

4.3.2. ETS. In Figure 6, the ETS values calculated for several rainfall thresholds at 24,48 , and $72 \mathrm{~h}$ of forecast are shown. This index gives a measure of the frequency of correctly predicted events. The reported value excluded a number of cases for which a correct forecast was performed by chance (according to (2)). It can be concluded that the NTHF always presented better ETSs than the R-CLIPER. The best predictions were obtained for thresholds ranging from 6.4 to $51 \mathrm{~mm}$, for which the ETS was approximately 0.4 . For heavy rainfall events, the ETS decreased, indicating the difficulty in forecasting large rainfall volumes for both models. In particular, the R-CLIPER provides an average description of the climatological precipitation, and it was unsuitable for the description of heavy rainfall. In both systems, the ETS was low for lighter rain.

\subsection{Mean Rainfall and Rain Flux Distribution}

4.4.1. Radial Distribution. The mean radial profiles for $24 \mathrm{~h}$ forecasts of the TRMM, NTHF, and R-CLIPER are shown in Figure 7(a). In Figure 7(b), the bias, defined as the difference between the predictions and the measurements, is represented. The radii considered were in the interval $30 \mathrm{~km} \leq R \leq 600 \mathrm{~km}$. In Figures S2 and S3 of the 


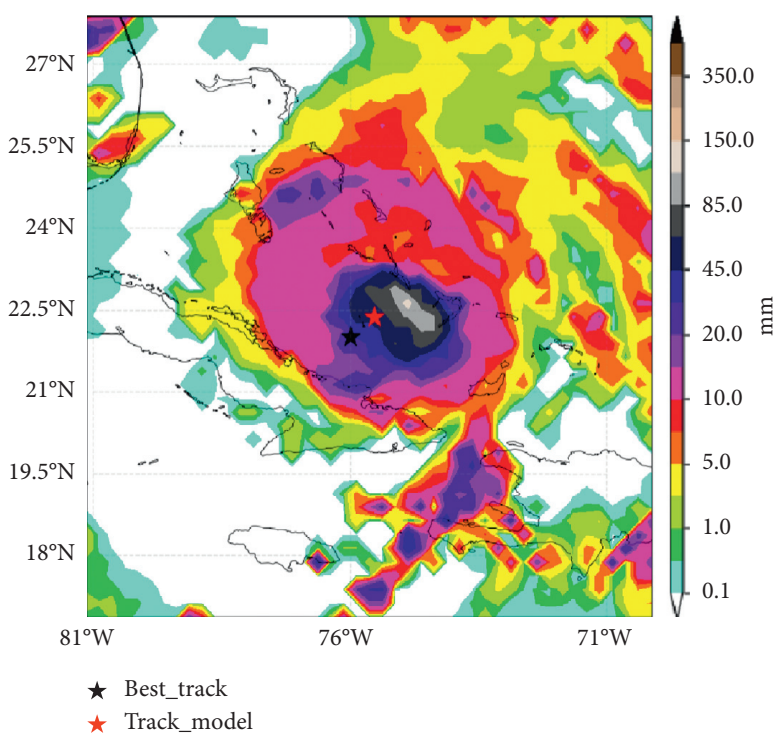

(a)

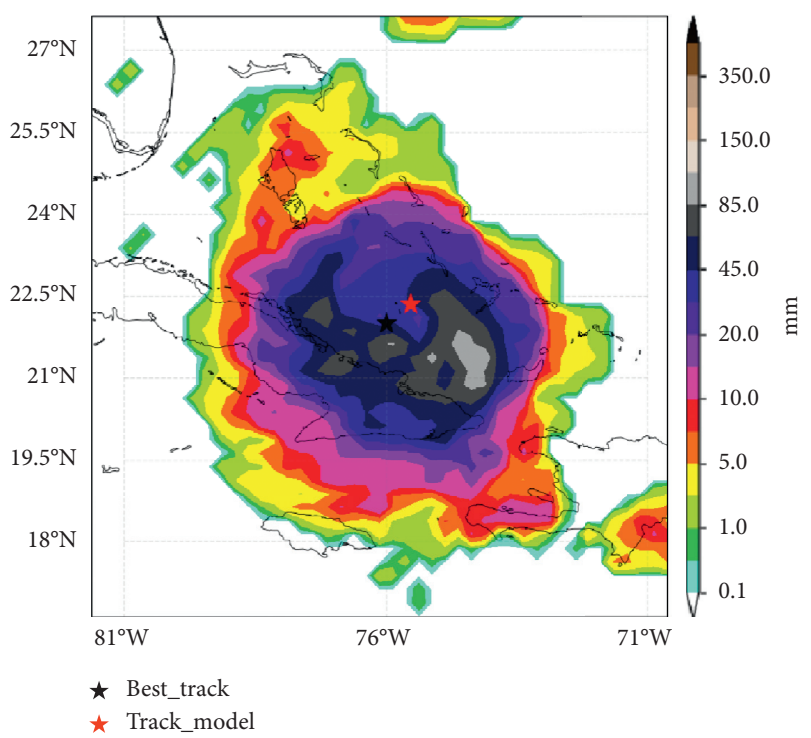

(b)

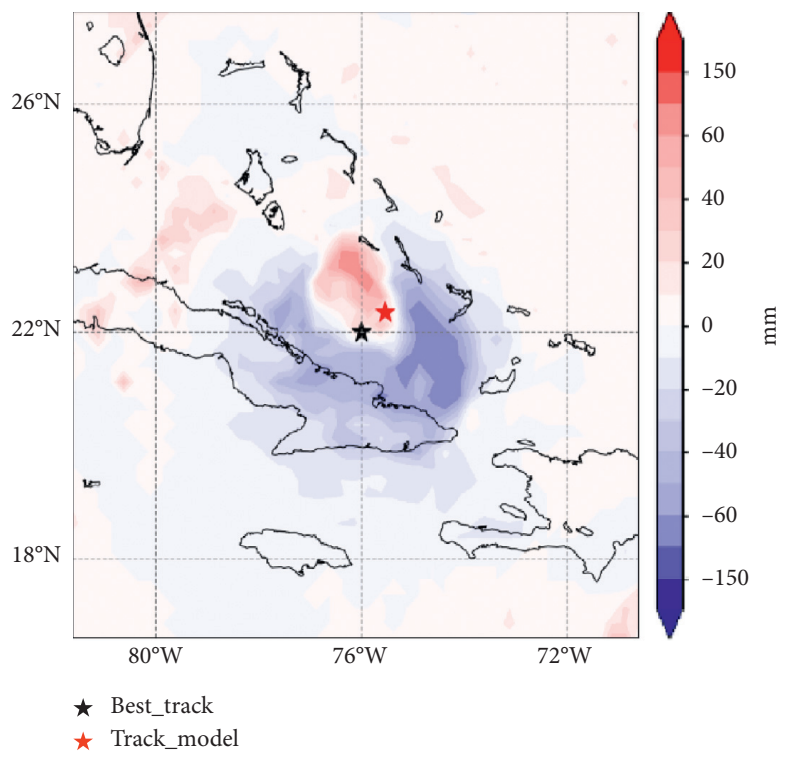

(c)

Figure 4: Comparison between NTHF prediction and TRMM. (a) Accumulated precipitation in first six hours, predicted by NTHF for Hurricane Irma at 1800 UTC, September 8, 2017. (b) Accumulated precipitation obtained from TRMM for the same hurricane, date, and time. (c) Difference between both graphics, providing bias values from the grid points. Stars represent the centre of the storm as the best track (black star) and NTHF forecast (red star).

Supplementary Materials, the values for 48 and $72 \mathrm{~h}$ forecasts are shown. Both models showed the same decrement in the predicted rainfall with the distance from the centre of the storm. The predictions of the R-CLIPER underestimated the rainfall distribution in all the profiles, in which the larger bias $(20-50 \mathrm{~mm})$ was close to the core of the storm. The bias was almost zero in the periphery $(500-600 \mathrm{~km}$ ) of the cyclone. These results were identical for all forecast times.

Up to $24 \mathrm{~h}$, the NTHF overestimated the observed rainfall by $10 \mathrm{~mm}$ from the centre of the storm up to a radius of $320 \mathrm{~km}$, with a negligible bias at larger radius. This is attributable to the use of the Ferrier-Aligo parameterization scheme. Wang and Phillips [39] demonstrated that this scheme generated less stratiform clouds and less anvil clouds out of the eye wall owing to the lower heating ratio in this zone. In particular, it provoked the maximum rainfall in the region from the centre to the eye wall. Additionally, the use of convective parameterization with a low horizontal resolution $(\sim 27 \mathrm{~km})$ further activated the convection in the region close to the centre. All these factors were reflected in the modelling of rain accumulates, surpassing the values detected by the TRMM. Ko et al. [40] modelled the precipitation provoked by Hurricane Harvey and obtained an overestimation of the rainfall (predicted by the HWRF) when compared with gauge data up to a radius of $120 \mathrm{~km}$. 


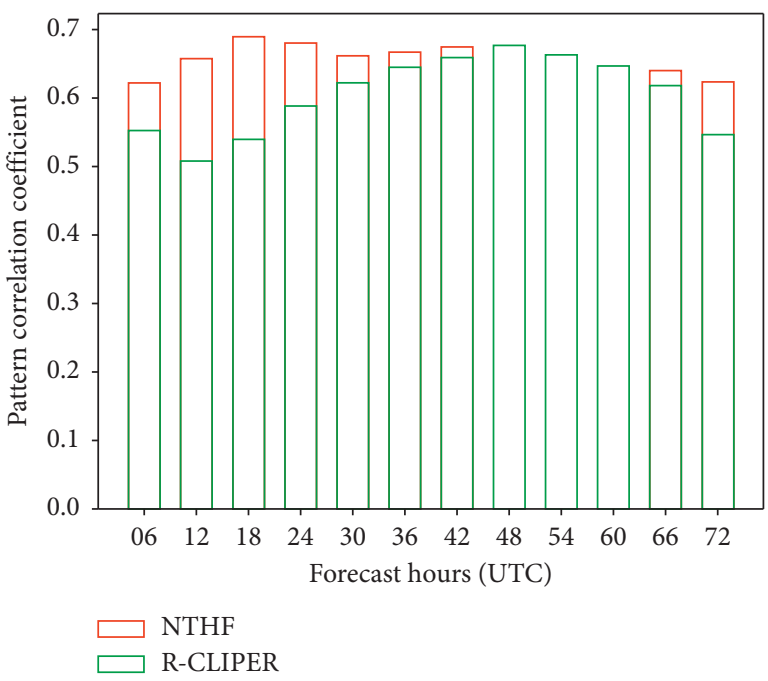

Figure 5: Pattern correlation coefficients. Time evolution of correlation coefficient between observations of TRMM and forecasts performed by NTHF (red bars) and R-CLIPER (green bars) is shown. Both forecasts improved with time and exhibited a final decrement. In general, NTHF performed better than R-CLIPER.

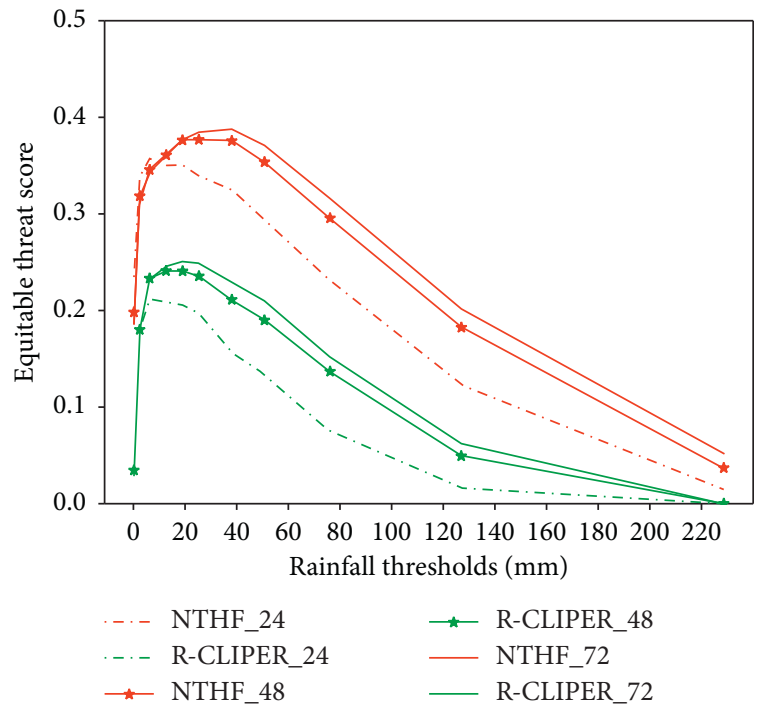

Figure 6: Equitable threat score (ETS) for both systems. Dependence of ETS with rainfall thresholds at three forecast times. NTHF always performs better than R-CLIPER.

At $48 \mathrm{~h}$, the NTHF system underestimated the precipitation from 0 to $130 \mathrm{~km}$, whereas it overestimated it for all the other radii, although the amount was less than $10 \mathrm{~mm}$. For $72 \mathrm{~h}$, an underestimation (less than $20 \mathrm{~mm}$ ) in the central zone of the cyclone was detected, whereas a behaviour similar to that of the TRMM was observed for larger distances from the centre.

4.4.2. Rain Flux. The forecast of the total volume of water deposited by rain associated with a cyclone in a specified region is important because this volume is related with the possibility of flooding. Rain flux is often used as an indicator to compare between models that use different grid areas. It is defined as the product of rainfall in a specified grid point and the area represented by the grid point $\left(\sim 27.27 \mathrm{~km}^{2}\right.$ in this study). It can be expressed in units of $\mathrm{mm} \cdot \mathrm{km}^{2}$, or in $\mathrm{km}^{3}$. Furthermore, it is proportional to the rainfall amount, thereby enabling one to use the volume of water deposited by rain (the rain volume) instead of the number of times a specific threshold has been exceeded [7].

Figure 8(a) shows the total rain flux estimated by the TRMM and calculated by the NTHF and R-CLIPER as a function of forecast time up to $72 \mathrm{~h}$. The bias, determined as the difference between the forecast and TRMM measurement, is shown in Figure 8(b). Both figures reveal the quality of the NTHF forecast of total rain volume, underestimating no more than $5 \mathrm{~km}^{3}(\approx 4.4 \mathrm{~mm})$ in the first $42 \mathrm{~h}$ and overestimating a maximum value of $10 \mathrm{~km}^{3}(\approx 8.8 \mathrm{~mm})$ in the final hours of the forecast.

4.4.3. Mean Rainfall Statigraphs. To further characterise the mean rainfall (shown in Figure 7), we calculated the BIAS, MAE, and RMSE (as shown in (4)-(6), respectively) for every grid point. Figure 9 shows the BIAS of both models compared with TRMM. It is clear that the NTHF always had a smaller BIAS than the R-CLIPER, underestimating the mean rainfall until 42 hours of the forecast. The BIAS in this time interval was smaller than $3 \mathrm{~mm}$. Beyond $42 \mathrm{~h}$, an overestimation occurred, with maximum values less than $9 \mathrm{~mm}$. The R-CLIPER always underestimated the rainfall, with a maximum BIAS of $25 \mathrm{~mm}$.

To evaluate the average disagreement between the forecast and the rainfall measured by the TRMM, the MAE was calculated (see (4)). Figure 10 shows the time evolution of the MAE for the NTHF and R-CLIPER. It is clear that the NTHF (to a forecast time of $60 \mathrm{~h}$ ) had a smaller MAE than the R-CLIPER, i.e., less than $20 \mathrm{~mm}$ up to $30 \mathrm{~h}$. For the forecast time beyond $60 \mathrm{~h}$, both systems performed almost equally. Comparing Figures 9 and 10, it can be concluded that the R-CLIPER underestimated the mean rainfall in almost every grid point, whereas the NTHF underestimated it in some grid points and overestimated it in others, thereby yielding a bias that was much smaller than that of the R-CLIPER; however, the MAE was almost equal for both systems.

Another measure of the forecast quality is the RMSE (see (5)). Figure S4 of the Supplementary Materials shows a graph with the time dependence of RMSE for both systems. For time intervals below $48 \mathrm{~h}$, the NTHF performed better. For ulterior forecast times, this statigraph indicated a better performance of the R-CLIPER. Figures 10 and S4 provide similar results and indicated similar dependence.

4.4.4. PDF and CDF. The PDF provides the frequency of rainfall occurrence pertaining to different rain thresholds. It was calculated for the observations and the two forecast systems. Figure 11(a) shows the PDF for the first $24 \mathrm{~h}$ of forecast. Using this data, the CDF, which provides the percentile at which a specified rain threshold is reached, can be calculated. 


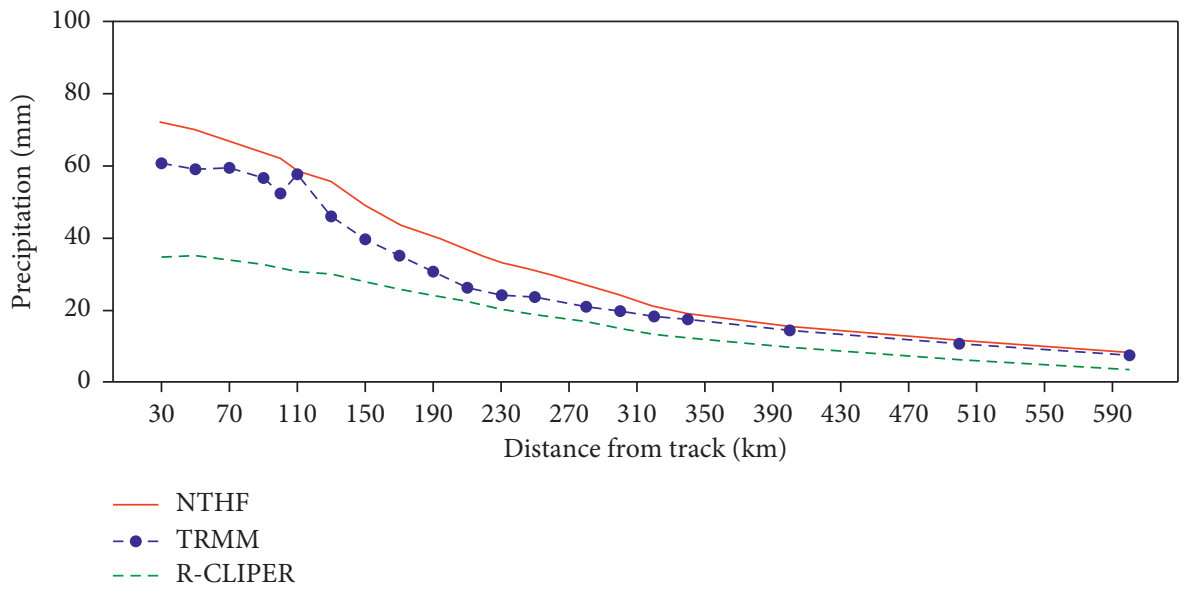

(a)

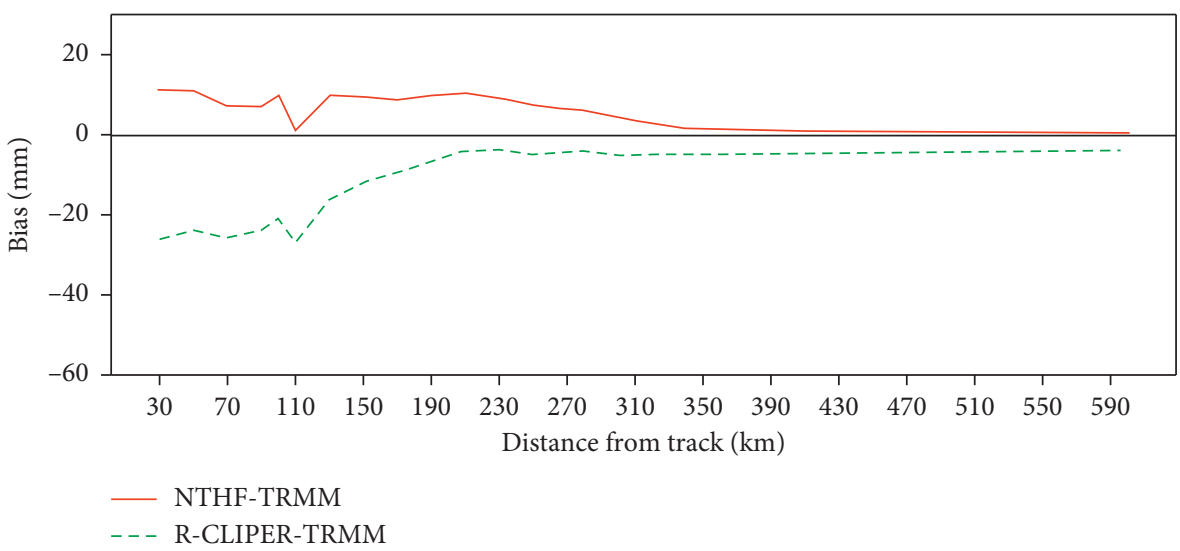

(b)

FIgURE 7: Mean rainfall radial distribution at $24 \mathrm{~h}$. (a) Values determined from TRMM and calculated by NTHF and R-CLIPER. (b) Bias between system predictions and TRMM measurements.

In particular, the 50th percentile provides a threshold above which $50 \%$ of the rain occurs. It is typically assumed that the low-to-moderate rainfall zone appears below this percentile. If the observations indicate a specified 50th percentile threshold and the system forecasts a larger than 50th percentile, it indicates that the system is forecasting less rain in the low-to-moderate amount. Figure 11(b) shows the $\mathrm{CDF}$ for the first $24 \mathrm{~h}$ of forecast.

As shown in Figure 11(a), the maximum of the observed frequencies is in the thresholds between 10 and $31.6 \mathrm{~mm}$, consistent with the NTHF prediction. The R-CLIPER shows a maximum in the frequencies between 3.2 and $10 \mathrm{~mm}$. This is reflected in the values of the 50th percentile determined from Figure 11(b) (TRMM, $12.6 \mathrm{~mm}$; NTHF, $6.3 \mathrm{~mm}$; and R-CLIPER, $3.2 \mathrm{~mm}$ ). These values imply that the NTHF overestimated the light rain, whereas the R-CLIPER overestimated those values.

When forecast was performed in a $48 \mathrm{~h}$ interval (see Figure S5 in the Supplementary Materials), the same behaviour was observed, except that the difference for the $\mathrm{R}$-CLIPER was greater. The maximum frequencies for the TRMM, NTHF, and R-CLIPER were in the intervals $31.6-100 \mathrm{~mm}, 10-31.6 \mathrm{~mm}$, and $3.2-10 \mathrm{~mm}$, respectively. Meanwhile, their median values were $15.8,10$, and $5 \mathrm{~mm}$, respectively. For $72 \mathrm{~h}$ (see Figure S6 in the Supplementary Materials), the maximum frequencies for the TRMM, NTHF, and R-CLIPER were in the intervals $31.6-100 \mathrm{~mm}$, $10-31.6 \mathrm{~mm}$, and $3.2-10 \mathrm{~mm}$, respectively. Meanwhile, the median cumulative frequencies were $20.0,15.8$, and $5.0 \mathrm{~mm}$, respectively, consistent with the tendency shown above.

4.4.5. PDF and CDF of Rain in Bands. When the frequency distribution was segmented in bands around the centre of the forecast and observed track, the rainfall distribution was seen with more details, and the effect of the track error on the analysis of the precipitation forecast can be decreased. The total rainfall was segmented into circular bands of $100 \mathrm{~km}$ radius. Among those bands, the innermost one $(0$ to $100 \mathrm{~km}$, including the eyewall) presents the heavier precipitation, whereas the outer bands include stratiform zones and rainbands [7]. In Figures S7-S9 of the Supplementary Materials, the PDF and CDF for the band between 0 and $100 \mathrm{~km}$ for 24,48 , and $72 \mathrm{~h}$ are shown. The PDF and CDF of the band between 300 and $400 \mathrm{~km}$ are represented in Figures S10-S12 of the Supplementary Materials for the same forecast time, respectively. For the innermost band, the forecast and observations for light rain (rainfall threshold 

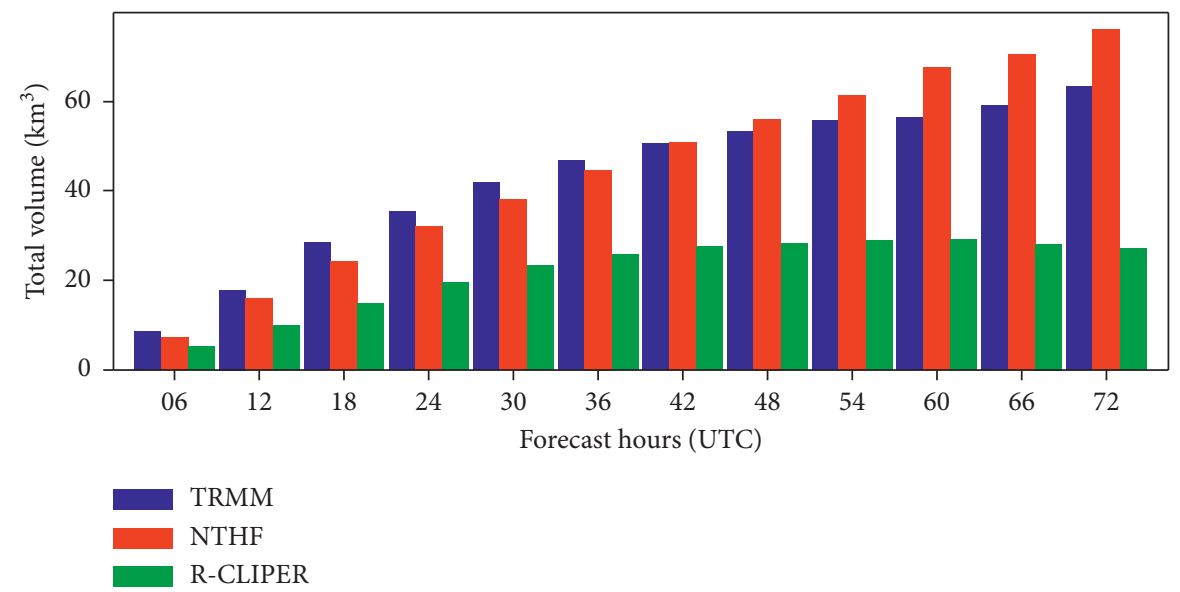

(a)

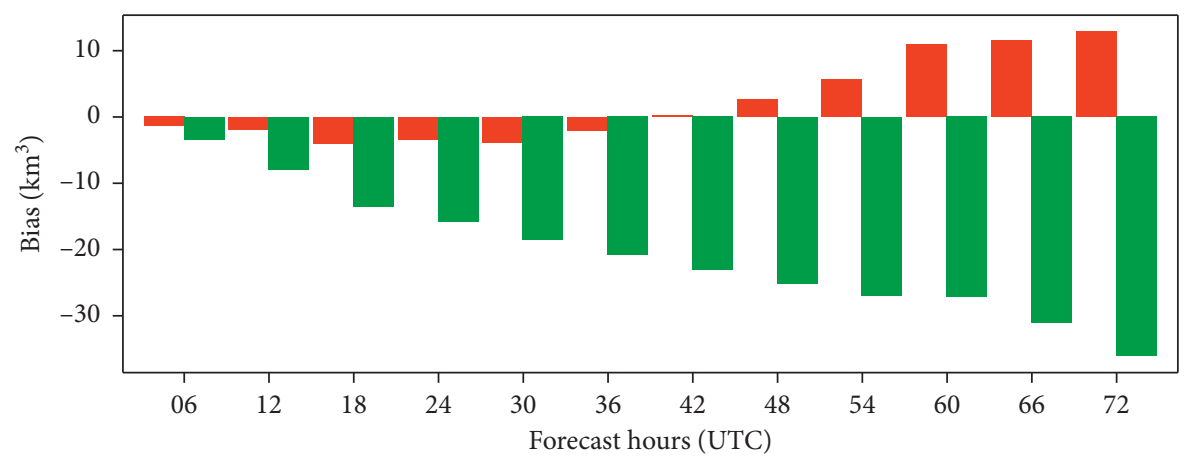

NTHF-TRMM

R-CLIPER-TRMM

(b)

Figure 8: Total rain volume at different times. (a) Values determined from TRMM and calculated by NTHF and R-CLIPER. (b) Bias between system predictions and TRMM measurements.

below $10 \mathrm{~mm}$ ) agreed well with each other. For values above $31.6 \mathrm{~mm}$, the NTHF presented better agreement with the observations, where it only overestimated heavy precipitation and underestimated light rainfall. The R-CLIPER performed well in this band, although it underestimated the large rainfall. In the 300-400 km band, the NTHF correctly described the rainfall distribution for all forecast times and for most of the thresholds. The R-CLIPER indicated a significant bias in the interval from 1 to $10 \mathrm{~mm}$, overestimating the small amounts and underestimating the remaining thresholds.

Figure 12 summarises the thresholds, showing the maximum frequency for the different bands at 24 (Figure 12(a)), 48 (Figure 12(b)), and $72 \mathrm{~h}$ (Figure 12(c)). The NTHF overestimated the heavy rain in the central part of the storm $(0-100 \mathrm{~km}$ band). In the $24 \mathrm{~h}$ forecast, it underestimated the rain in the $100-200 \mathrm{~km}$ zone and overestimated the rain in the outer zones of the cyclone. In the 48 and $72 \mathrm{~h}$ forecast by the NTHF, the rainfall threshold in the outer zone was overestimated and the mid radii was underestimated. Meanwhile, the R-CLIPER underestimated the observed maximum frequency thresholds.
4.5. Extreme Rain Prediction. To evaluate the efficacy of a system in describing the extreme rain amount, the threshold for which the observed precipitation has a 95\% cumulative frequency (95th percentile) in the CDF must be determined. Subsequently, from the CDF predicted by the system, the percentile corresponding to a rain amount equal to the $95 \%$ value of the observations is to be extracted. If the percentage is smaller than $95 \%$, it means that the system overestimates the extreme rain amounts. On the contrary, if the percentage is larger than $95 \%$, the system underestimates the amount of extreme rain.

From the CDF of the $0-100 \mathrm{~km}$ band, which contains the zone with larger precipitations, the NTHF behaved similarly to that observed for $24 \mathrm{~h}$; however, for 48 and $72 \mathrm{~h}$, it overestimated the part of the precipitation corresponding to large accumulates. The R-CLIPER could not forecast large rain amounts because the threshold corresponding to $95 \%$ of the observed cumulative frequencies corresponded to $100 \%$ of the cumulative frequency calculated by the system, implying that it cannot predict extreme rain events. 


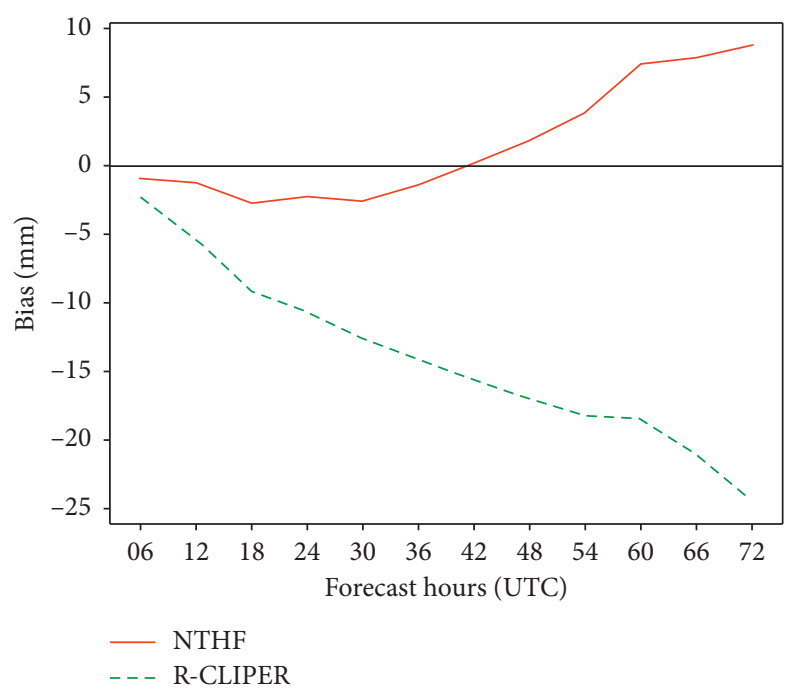

FIGURE 9: Time evolution of bias for mean rainfall. Bias, calculated as the difference between the mean rainfall predicted by NTHF (red line) and R-CLIPER (green line) and TRMM measurements, as a function of forecast hour.

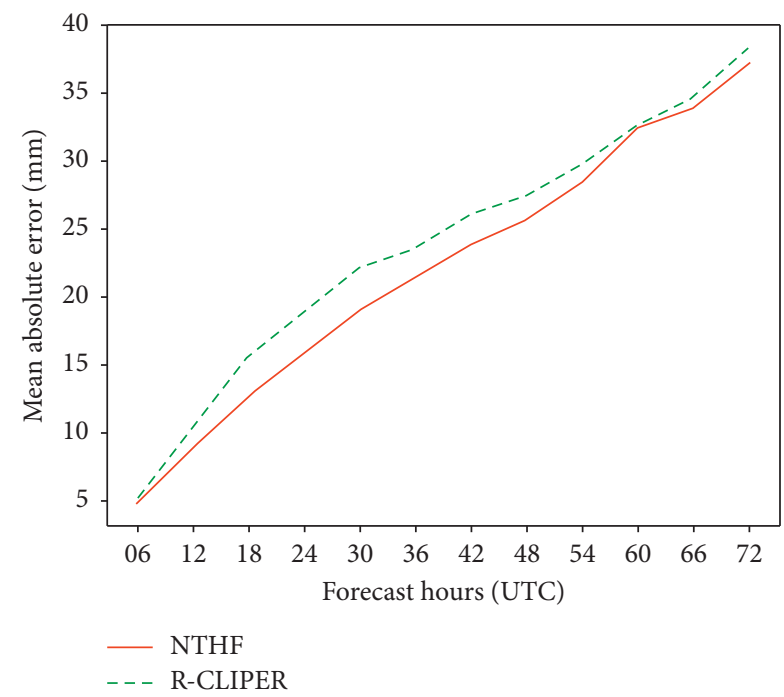

FIgURE 10: The time evolution of the mean absolute error of both systems. It is possible to note the better performance of NTHF (red line) compared with R-CLIPER (green line).

4.6. Evaluation of Forecast Skills. Using the data presented above, the ability indices associated with the NTHF and R-CLIPER were calculated. Using the obtained values, we assessed the capabilities of the NTHF as an operative tool in the forecast of rainfall provoked by TCs in the NATL, particularly in the Caribbean Sea and the Gulf of Mexico.

4.6.1. Pattern Matching. The NTHF system can represent large-scale rain patterns effectively (when compared with the $\mathrm{R}$-CLIPER). The values of this ability index were between 0.4 and 0.5 . These values are related with the high average values of the correlation coefficient $(\sim 0.6)$, and the average values of the ETS was approximately 0.3 . These values were similar to those obtained in [7] for three dynamical models. The values for the R-CLIPER were between 0.2 and 0.4 , which were determined by the low values of the ETS $(\sim 0.15)$. This poor performance may be associated with the asymmetry in the rain distribution of the cyclones considered in the sample. These asymmetries can be predicted by the numerical model because the parameterizations used (involving microphysics and convection processes) yielded a close agreement between the model and reality. Regarding the climatological model, the assessment of the rain amounts was based on the symmetric distribution of rain, which deviate significantly from reality. For the larger radii, although the R-CLIPER estimates were closer to the TRMM output, the ETS was extremely low, resulting in a low ability for predicting the local rain distribution.

The values of pattern matching depended significantly on the track forecast error. In [18], it was demonstrated that the NTHF can forecast the cyclone track in the first $48 \mathrm{~h}$, similar to the error reported by the NHC in its official forecast until 2016. Therefore, the ETS of the NTHF and GFDL were similar (see [7]). For the $72 \mathrm{~h}$ forecast, the value was of 0.48 , which was similar to the evaluation of GFDL by Marchok et al. [7], i.e., 0.46. The inclusion of vortex relocation in the numerical system will improve this ability.

4.6.2. Average Rainfall and Its Distribution. The NTHF performed better than the R-CLIPER in forecasting the mean rain. This is due to its superior performance in the two indices that compose the mean rain skill index. Its MREI value was $\sim 0.9$, larger than that of the R-CLIPER $(\sim 0.7)$. Furthermore, its CDF - MVI, related with the central value in the rainfall distribution, was $\sim 0.7$, whereas that for the $\mathrm{R}$-CLIPER was only 0.5 . These results indicate that, although the R-CLIPER represented the average radial distribution of rain effectively, it presented limitations in systems with uneven radial distributions of rain in different directions, which are associated with the occurrence of bands with intense rain and zones of almost no rain, particularly at radii below $400 \mathrm{~km}$. These asymmetries caused the R-CLIPER to predict unrealistic large areas with extremely light rainfall, whereas the dynamical models reproduced the asymmetries. For instance, the values obtained by the NTHF (0.8-0.9) highlight the correct representation of rainfall for this system, even for cyclones with conditions that generate large asymmetries due to interactions with frontal bands, a complex topography, and/or wind shear. The obtained values compared well with the value (0.74) calculated in [7] for GFDL. The abovementioned factor also resulted in the higher ETS for the NTHF compared with the R-CLIPER.

The predicted quality of rain volume in the observation zone (radius $600 \mathrm{~km}$ around the track centre) was similar to that obtained by Marchok et al. [7] for $72 \mathrm{~h}$ using the GFDL model. The bias of $10 \mathrm{~km}^{3}$ in this study was close to the value of $9.4 \mathrm{~km}^{3}$ obtained in [7]. It is noteworthy that for models GFS $\left(5.2 \mathrm{~km}^{3}\right)$ and NAM $\left(0.9 \mathrm{~km}^{3}\right)$, the bias was smaller, which coincided with the results of Brennan et al. [41]. In this study, TCs from 2005 to 2007 were investigated; it was 


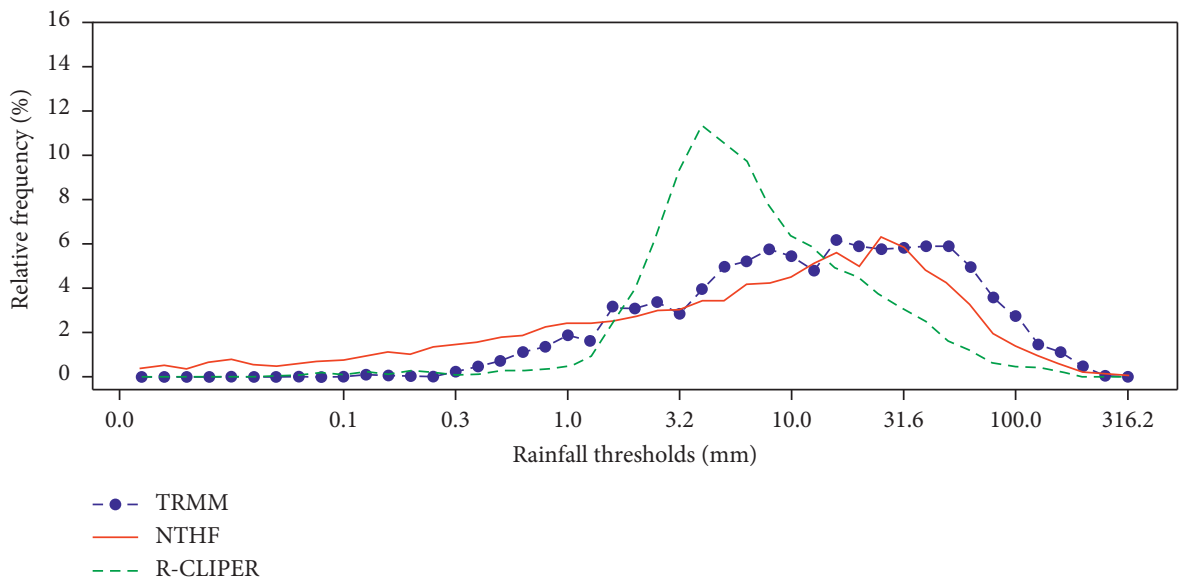

(a)

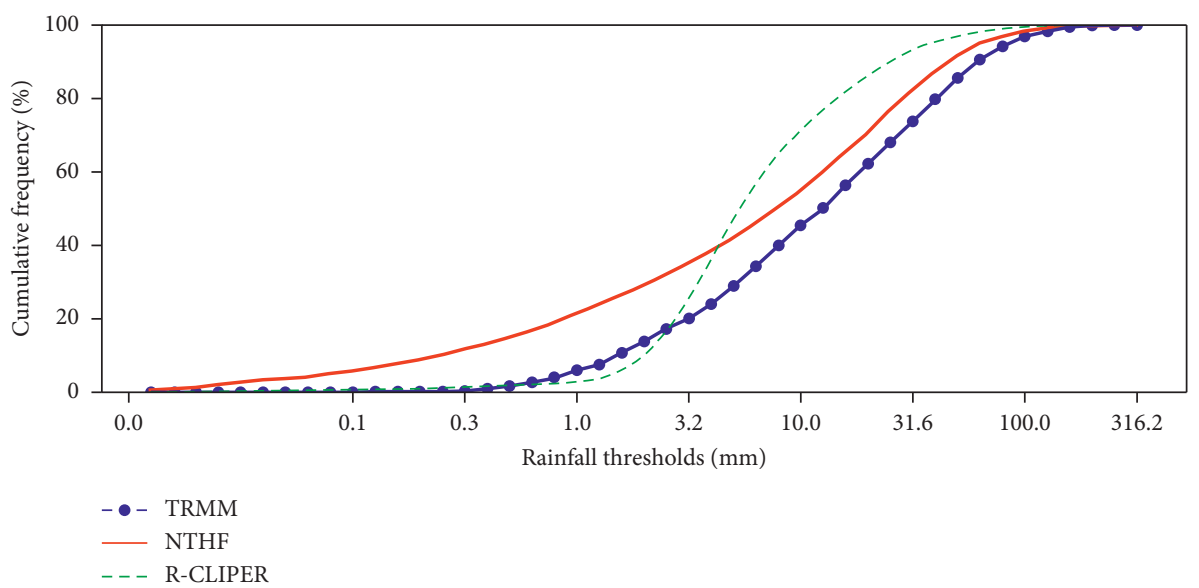

(b)

FIGURE 11: Frequency distribution of rain flux in first $24 \mathrm{~h}$ of forecast. (a) Probability distribution function of observed (blue line) frequency of rainfall above a specified threshold, as well as that of NTHF (red line) and R-CLIPER (green line). (b) Cumulative distribution function of observed and predicted data, calculated from frequency distribution data.

discovered that the NAM presented a small bias but indicated limitations in predicting extreme amounts of rain. Furthermore, it was discovered that the GFS and the European model ECMWF (European Centre for MediumRange Weather Forecasts) performed well.

The values of the MAE and BIAS for the mean rainfall were similar to those of Tuleya et al. [42]. An average BIAS of $8.3 \mathrm{~mm}$ and an MAE of $23 \mathrm{~mm}$ at $72 \mathrm{~h}$ of forecast were obtained using the GFDL for 25 TCs. The R-CLIPER (see Figure 8(b)) underestimated the total volume, reaching a maximum BIAS of $32 \mathrm{~km}^{3}$ at $72 \mathrm{~h}$. The reasons for the divergence are explained above. The differences in the distribution for the NTHF (overestimating the frequency in the smaller thresholds) is a limitation inherited from the HWRF model, a deficiency associated with the cumulus parameterization. Additionally, it is clear that the NTHF overestimated the maximum in the $0-100 \mathrm{~km}$ band (around the eyewall) compared with the observations. The R-CLIPER underestimated the frequencies in all bands, in which larger differences were discovered in radii up to $400 \mathrm{~km}$ owing to the large asymmetries observed. For radii above $400 \mathrm{~km}$, where the rain distribution was more uniform, the differences were smaller. It is clear from Figure 12 that the maximum threshold decreased with the radius, and a radial profile exhibiting the behaviour obtained by Lonfat et al. [6] was obtained.

It is important to control these results comparing the TRMM observations with gauge and radar data; this is because it has been reported $[14,27]$ that rainfall satellite estimates (particularly TRMM) presented biases when compared with gauge data. The final assessment of the NTHF bias involves these comparisons.

4.6.3. Extreme Rain Amount. By comparing the threshold value of the 95th percentile in the observation with the percentile corresponding to this value for the forecast and by calculating the maximum value index, it can be concluded that the NTHF can accurately predict (MVI 1.0) extreme rain for a $24 \mathrm{~h}$ forecast. For 48 and $72 \mathrm{~h}$ forecasts (see Figures S8 and S9, Supplementary Materials), the values of extreme rain calculated by the model were approximately 9-11\% in the CDF, which is a large overestimation, mainly in the $1-100 \mathrm{~km}$ band. This is attributable to the increase in the 


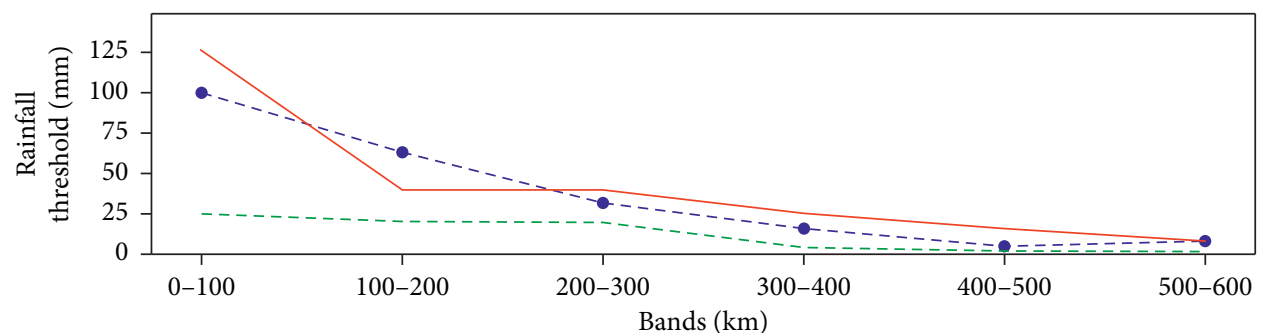

- TRMM

NTHF

- - R-CLIPER

(a)

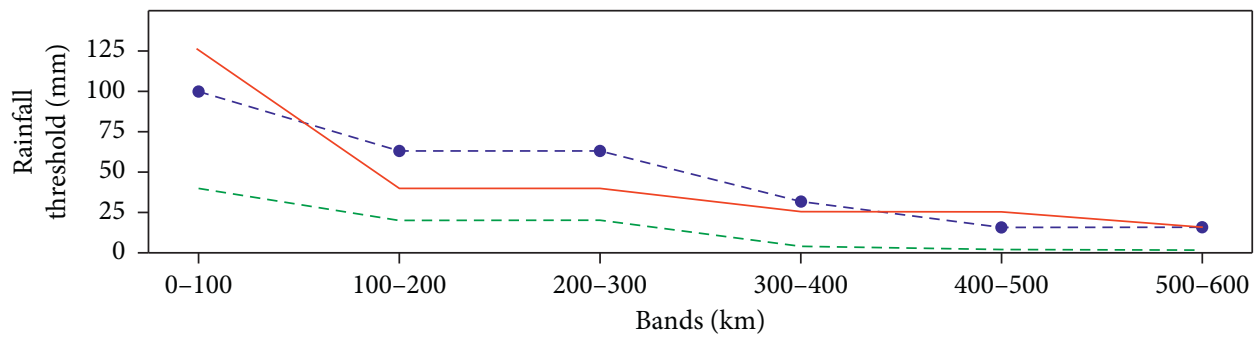

$-\bullet-$ TRMM

- NTHF

- - R-CLIPER

(b)

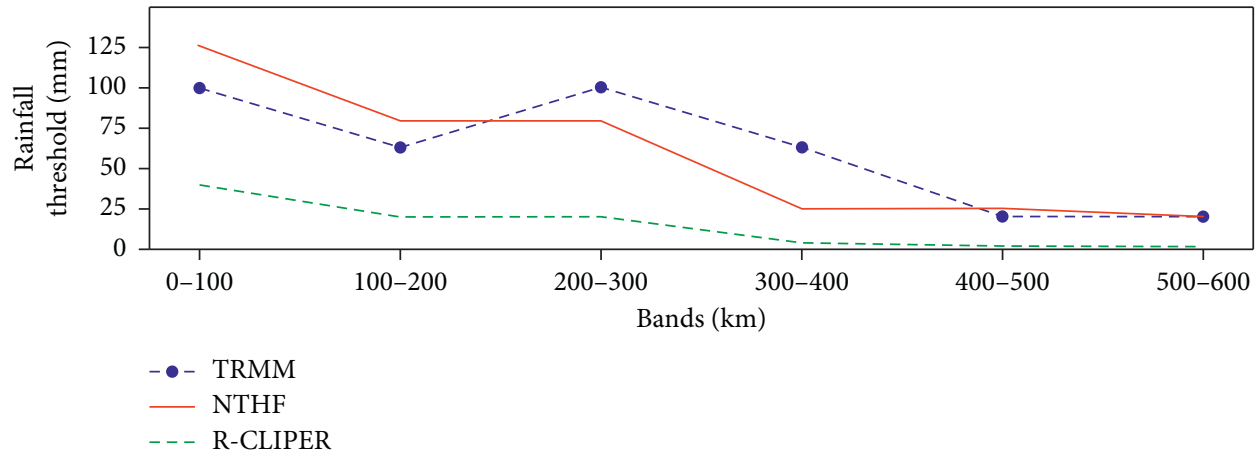

(c)

FIGURE 12: Rainfall thresholds at which maximum frequency appeared for different bands. This figure summarises the results for the TRMM (blue), NTHF (red), and R-CLIPER (green) for (a) $24 \mathrm{~h}$, (b) $48 \mathrm{~h}$, and (c) $72 \mathrm{~h}$ forecasts.

track forecast error for the sample used in this study (see Figure 3) and also the representation of microphysics models in the resolution used [7]. These results were similar to those in [43], which reported an overestimation of approximately $8 \%$ rainfall in the vicinity of the storm core. For these time intervals, the system did not perform well. In the future, the innermost domain $(9 \mathrm{~km})$ of the NTHF will be evaluated to achieve a better representation of rain generating processes, associated particularly with extreme rains.

\section{Conclusions}

The NTHF system was evaluated to be used in the QPF associated with TCs. It demonstrated the best performance up to a $24 \mathrm{~h}$ forecast. Its performance was of the same quality as that of other numerical systems reported in the literature.
Compared with the TRMM, the system underestimated the rain volume up to $42 \mathrm{~h}$ and overestimated it in the subsequent hours. At $24 \mathrm{~h}$, the MAE and RMSE were 16 and $27 \mathrm{~mm}$, respectively, both of which increased with time. The indices of the QPF were approximately 0.48 for determining the rain pattern and $0.80-0.90$ for the mean rain and rain flux distribution at all the forecast hours, similar to the results of a previous study. Regarding the prediction of extreme rain, it only performed well for $24 \mathrm{~h}$. In future studies, we plan to perform data assimilation to improve the precision in the beginning of the observation period. Furthermore, we plan to perform a hot start in the system initialisation. Additionally, we will include vortex relocation in the model to obtain the rain values, assuming that the simulated field coincides with the real field. Comparisons with real gauge and radar data will be performed to evaluate 
the local performance of the system. Finally, we will perform comparisons with other precipitation reanalysis databases, such as NARR, CFSR, and ERA5 for a greater generalisation of the results.

\section{Data Availability}

The data used in this article to carry out the validation are public and are available at http://www.pmm.nasa.gov/dataaccess/downloads/trmm.shtml. The outputs of the NTHF model can be reproduced by performing the simulations with the initialisation data. The outputs of the GFS at $0.5^{\circ}$ resolution are obtained from https://nomads.ncdc.noaa.gov/ data/gfs4/. Finally, the actual tropical cyclone trajectories are public and they are available at https://www.nhc.noaa.gov/ data/\#hurdat.

\section{Conflicts of Interest}

The authors declare that there are no conflicts of interest regarding the publication of this paper.

\section{Acknowledgments}

The authors acknowledge the availability of public datasets from the Tropical Rainfall Measuring Mission, the National Hurricane Centre, and the Global Forecasting System.

\section{Supplementary Materials}

The following are available in Supplementary Materials: Figure S1, Figure S2, Figure S3, Figure S4, Figure S5, Figure S6, Figure S7, Figure S8, Figure S9, Figure S10, Figure S11, and Figure S12. (Supplementary Materials)

\section{References}

[1] E. N. Rappaport, "Loss of life in the United States associated with recent atlantic tropical cyclones," Bulletin of the American Meteorological Society, vol. 81, no. 9, pp. 2065-2073, 2000.

[2] E. N. Rappaport, "Fatalities in the United States from atlantic tropical cyclones: new data and interpretation," Bulletin of the American Meteorological Society, vol. 95, no. 3, pp. 341-346, 2014.

[3] S. R. Pérez, G. R. Vega, and M. Limia, "Los huracanes más intensos y desastrosos de Cuba en los últimos dos siglos," Boletín de Sometcuba, vol. 7, 2001.

[4] L. J. Shapiro, "The asymmetric boundary layer flow under a translating hurricane," Journal of the Atmospheric Sciences, vol. 40, no. 8, pp. 1984-1998, 1983.

[5] M. A. Bender, "The effect of relative flow on the asymmetric structure in the interior of hurricanes," Journal of the Atmospheric Sciences, vol. 54, no. 6, pp. 703-724, 1997.

[6] M. Lonfat, F. D. Marks, and S. S. Chen, "Precipitation distribution in tropical cyclones using the tropical rainfall measuring mission (TRMM) microwave imager: a global perspective," Monthly Weather Review, vol. 132, no. 7, pp. 1645-1660, 2004.

[7] T. Marchok, R. Rogers, and R. Tuleya, "Validation schemes for tropical cyclone quantitative precipitation forecasts: evaluation of operational models for U.S. landfalling cases," Weather and Forecasting, vol. 22, no. 4, pp. 726-746, 2007.

[8] J. Zawislak, H. Jiang, G. R. Alvey et al., "Observations of the structure and evolution of hurricane Edouard (2014) during intensity change. Part I: relationship between the thermodynamic structure and precipitation," Monthly Weather Review, vol. 144, no. 9, pp. 3333-3354, 2016.

[9] J. L. Franklin, C. J. McAdie, and M. B. Lawrence, "Trends in track forecasting for tropical cyclones threatening the United States, 1970-2001," Bulletin of the American Meteorological Society, vol. 84, no. 9, pp. 1197-1204, 2003.

[10] M. DeMaria, M. Mainelli, L. K. Shay, J. A. Knaff, and J. Kaplan, "Further improvements to the statistical hurricane intensity prediction scheme (SHIPS)," Weather and Forecasting, vol. 20, no. 4, pp. 531-543, 2005.

[11] M. DeMaria and R. Tuleya, "Evaluation of quantitative precipitation forecasts from the GFDL hurricane model," in Reprints Symposium on Precipitation Extremes: Predictions, Impacts, and Responses, pp. 340-343, American Meteorological Society, Boston, MA, USA, 2001.

[12] F. D. Marks and M. DeMaria, "Development of a tropical cyclone rainfall climatology and persistence (R-CLIPER) model," Technical report, Australian Centre for International Agricultural Research, Canberra, Australia, 2003.

[13] S. Bhomia, N. Jaiswal, and C. M. Kishtawal, "Accuracy assessment of rainfall prediction by global models during the landfall of tropical cyclones in the north Indian ocean," Meteorological Applications, vol. 24, no. 3, pp. 503-511, 2017.

[14] X. Zhang, E. N. Anagnostou, and H. Vergara, "Hydrologic evaluation of NWP-adjusted CMORPH estimates of hurricane-induced precipitation in the southern appalachians," Journal of Hydrometeorology, vol. 17, no. 4, pp. 1087-1099, 2016.

[15] L. M. Sierra, H. A. L. Ferrer, R. Valdés et al., "Sistema automático de prediccioón a mesoescala de cuatro ciclos diarios," Instituto de Meteorología, La Habana, Cuba, Informe de Resultado, 2015.

[16] B. A. Pérez, O. O. Díaz, and I. Mitrani, Sistema de Pronóstico Numérico Océano-Atmósfera Para la República de Cuba, VII Congreso Cubano de Meteorología, Havana, Cuba, 2013.

[17] B. A. Pérez, I. Mitrani, O. R. Díaz, C. Wettre, and L. H. Robert, "A numerical prediction system combining ocean, waves and atmosphere models in the Inter-American Seas and Cuba," Revista Cubana de Meteorología, vol. 25, pp. 109-120, 2019.

[18] A. P. Alarcón, "Sistema para el pronóstico numérico de la intensidad y trayectoria de ciclones tropicales en Cuba," M.S. thesis, Universidad de La Habana, La Habana, Cuba, 2019.

[19] V. B. Dodla, S. Desamsetti, and A. Yerramilli, "A comparison of HWRF, ARW and NMM models in hurricane Katrina (2005) simulation," International Journal of Environmental Research and Public Health, vol. 8, no. 6, pp. 2447-2469, 2011.

[20] D. Tran and S. Chiao, "Evaluating HWRF modeling of landfallling TCs over Florida," in Special Symposium on the Next Level of Predictions in Tropical Meteorology: Techniques, Usage, Support and Impacts, American Meteorological Society, Boston, MA, USA, 2013.

[21] Z. Janjic, T. Black, M. Pyle, B. Ferrier, and H. Y. Chuang, NMM Version 3 Modelling System User's Guide, Developmental Testbed Center/National Centers for Environmental Prediction, College Park, MD, USA, 2014.

[22] M. K. Biswas, L. Carson, K. Newman, L. Bernardet, E. Kalina, and E. Grell, "Community HWRF users' guide V3.9a," 2017.

[23] M. A. Bender and I. Ginis, "Real-case simulations of hurricane-ocean interaction using a high-resolution coupled 
model: effects on hurricane intensity," Monthly Weather Review, vol. 128, no. 4, pp. 917-946, 2000.

[24] Z. Yu and Y. Wang, "Rainfall distribution in landfalling TCs," in Extreme Weather, pp. 50-84, IntechOpen, London, UK, 2018.

[25] Y. Chen, E. Ebert, K. Walsh, and N. Davidson, "Evaluation of TRMM $3 B 42$ precipitation estimates of TC rainfall using PACRAIN data," Journal of Geophysical Research: Atmospheres, vol. 118, no. 5, pp. 1-13, 2013.

[26] H. Jiang, J. B. Halverson, and J. Simpson, "On the differences in storm rainfall from hurricanes Isidore and Lili. Part I: satellite observations and rain potential," Weather and Forecasting, vol. 23, no. 1, pp. 29-43, 2008.

[27] Z. Yu, H. Yu, P. Chen, C. Qian, and C. Yue, "Verification of tropical cyclone-related satellite precipitation estimates in mainland China," Journal of Applied Meteorology and Climatology, vol. 48, no. 11, pp. 2227-2241, 2009.

[28] W. Berg, T. L'Ecuyer, and C. Kummerow, "Rainfall climate regimes: the relationship of regional TRMM rainfall biases to the environment," Journal of Applied Meteorology and Climatology, vol. 45, no. 3, pp. 434-454, 2006.

[29] G. J. Huffman, D. T. Bolvin, E. J. Nelkin et al., "The TRMM multisatellite precipitation analysis (TMPA): quasi-global, multiyear, combined-sensor precipitation estimates at fine scales," Journal of Hydrometeorology, vol. 8, no. 1, pp. 38-55, 2007.

[30] Q. Sun, C. Miao, Q. Duan, H. Ashouri, S. Sorooshian, and K. L. Hsu, "A review of global precipitation data sets: data sources, estimation, and intercomparisons," Reviews of Geophysics, vol. 56, no. 1, pp. 79-107, 2018.

[31] B. Brown, E. Ebert, T. Fowler, E. Gilleland, P. Kucera, and L. Wilson, "Verification methods for TC forecasts," WMO Technical Document WWRP, vol. 7, 2013.

[32] N. S. Altman, "An introduction to kernel and nearestneighbor nonparametric regression," The American Statistician, vol. 46, no. 3, pp. 175-185, 1992.

[33] P. J. Englehart and A. V. Douglas, "The role of eastern north pacific tropical storms in the rainfall climatology of Western Mexico," International Journal of Climatology, vol. 21, no. 11, pp. 1357-1370, 2001

[34] J. Larson, Y. Zhou, and R. W. Higgins, "Characteristics of landfalling tropical cyclones in the United States and Mexico: climatology and interannual variability," Journal of Climate, vol. 18, no. 8, pp. 1247-1262, 2005.

[35] W. Zhang, G. Villarini, G. A. Vecchi, and H. Murakami, "Rainfall from tropical cyclones: high-resolution simulations and seasonal forecasts," Climate Dynamics, vol. 52, no. 9-10, pp. 5269-5289, 2019.

[36] M. F. Rios Gaona, G. Villarini, W. Zhang, and G. A. Vecchi, "The added value of IMERG in characterizing rainfall in tropical cyclones," Atmospheric Research, vol. 209, pp. 95-102, 2018.

[37] Anaconda Software Distribution, Computer Software, 2019, https://anaconda.com/.

[38] C. W. Landsea and J. L. Franklin, "Atlantic hurricane database uncertainty and presentation of a new database format," Monthly Weather Review, vol. 141, no. 10, pp. 3576-3592, 2013.

[39] Y. Wang and V. Phillips, "Evaluation and improvements of cloud and precipitation physics in the operational hurricane WRF model at NOAA/EMC," 2010.

[40] M.-C. Ko, F. D. Marks, G. J. Alaka Jr., and S. G. Gopalakrishnan, "Evaluation of hurricane Harvey (2017) rainfall in deterministic and probabilistic HWRF forecasts," Atmosphere, vol. 11, no. 6, p. 666, 2020.

[41] M. J. Brennan, L. J. Clark, and M. Klein, "Verification of quantitative precipitation forecast guidance from NWP models and the hydrometeorological prediction center for 2005-2007 tropical cyclones with continental U.S. rainfall impacts," in Proceedings of the AMS/WAF Conference, Orlando, FL, USA, April 2008.

[42] R. E. Tuleya, M. DeMaria, and R. J. Kuligowski, "Evaluation of GFDL and simple statistical model rainfall forecasts for U.S. landfalling tropical storms," Weather and Forecasting, vol. 22, no. 1, pp. 56-70, 2007.

[43] M. Ko, F. Marks, G. J. Alaka, and S. Gopalakrishnan, "Precipitation evaluation of the real-time basin-scale HWRF in 2017," in Proceedings of the 33rd Conference on Hurricanes and Tropical Meteorology, American Meteorological Society, Ponte Vedra Beach, FL, USA, April 2017. 